\title{
Pulsed Eddy Current: Feature Extraction Enabling In-Situ Calibration and Improved Estimation for Ferromagnetic Application
}

\author{
Faris Nafiah ${ }^{1,2}$ (D) Mohammad O. Tokhi ${ }^{1} \cdot$ Shiva Majidnia ${ }^{2} \cdot J^{\prime}$.hn Rudlin ${ }^{2} \cdot$ Zhanfang Zhao $^{1} \cdot$ Fang Duan $^{1}$
}

Received: 14 January 2020 / Accepted: 22 June 2020 / Published online: 30 June 2020

(c) The Author(s) 2020

\begin{abstract}
Steel pipes in process plant applications are often covered with insulation or weather protection that make inspection difficult because the additional layers need to be penetrated to inspect the pipes' structure. The pulsed eddy current (PEC) method was devised as a means of inspection through the surface layers. However, the performance of a PEC system is dependent on the electrical and magnetic properties of the pipe material, which are generally unknown. Therefore, the use of a calibration block from a different steel will give inaccurate results. The concept of calibrating using $\tau_{0}$ values obtained during inspection has undoubtedly been discussed in the literature. However, no comprehensive work was dedicated to using $|\nabla|^{-1}$ to carry out calibration on inspected structure. The linear relationship of the $|\nabla|^{-1}$ feature with the thickness squared, $d^{2}$, is first established using analytical solutions, and the calibration is carried out using the feature values obtained in air and the reference signal. The performance of this technique is assessed and compared with the conventional $\tau_{0}$ technique. Although both features exhibit similar immunity towards lift-off, $\tau_{0}$ technique requires normalisation procedure, which contributes to determining more configuration parameters. Experimental results also suggest the relative advantage of using $|\nabla|^{-1}$ feature in both wall thickness estimation and influences of noises.
\end{abstract}

Keywords Pulsed eddy current $\cdot$ Non-destructive testing $\cdot$ Feature extraction $\cdot$ Ferromagnetic application $\cdot$ Pipeline inspection

\section{Introduction}

Pipe inspection using pulsed eddy current (PEC) poses various challenges; in addition to the variation in the value of lift-off/insulation thickness, electromagnetic properties differ from one pipe to another. Therefore, in order to predict the output from a PEC inspection, the calibration sample should have exactly the same properties as the pipe to be inspected. For on-site inspection, this is rarely possible, and the errors introduced by the difference in materials is tolerated, as no practical alternative is available.

In most cases, to obtain a measure of the pipe thickness, the time at which the diffusion phase of the induced current ends, $\tau_{0}$, is employed. The relation between the wall thickness, $d$, and $\tau_{0}$ is claimed to be quadratic, i.e., $\tau_{0} \sim d^{2}[1-3]$,

Faris Nafiah

nafiahf@1sbu.ac.uk

1 School of Engineering, London South Bank University, London, UK

2 TWI Ltd, Cambridge, UK which indicates the ability to directly assess the wall thickness information from the aforementioned parameter.

The search for a better feature extraction technique can be seen in many previous works. One of the attempts is to make use of differential signal features, obtained by subtracting the test signals from the reference signal. The features extracted are claimed to be linear with wall thickness, although the presented results are limited to responses from analytical modelling [4].

An interesting implementation is reported by Huang et al. [5] expresses PEC signal as an infinite sum of exponentials, which enables the use of the fitted exponential coefficients as another feature for pipe thickness measurement. This paves the way to various other feature extraction techniques, such as obtaining the time derivative of the signal in logarithmic domain [6,7], exponential fitting [8], power law fitting technique [9], and a hybrid of Savitzky-Golay and adaptive least-square fitting (ALSF) [8]. These works, however, need either a calibration sample, or at least two known sample thicknesses to obtain the linearity function of the features with pipe wall thicknesses. Ulapane et al. [10] in particular have made use of the product of permeability and conduc- 
tivity to establish the calibration curve, which is therefore rather limited to pipes with known magnetic properties.

Deriving from the work of $[5,8,10,11]$, the work here proposes using PEC signal in air as an alternative to using calibration block. By comparing the time derivative of the PEC signals obtained in air and a reference zone on the inspected structure, a calibration curve can be derived. Thickness estimation can be achieved by making use of the calibration curve, without having to remove the insulation. The immunity of this feature towards lift-off provides significant advantage over conventional $\tau_{0}$ technique, which requires normalisation procedure to suppress the lift-off effects. The performances of $|\nabla|^{-1}$ and $\tau_{0}$ technique are also compared, using both simulation and experiments.

The rest of the paper is structured as follows: Sect. 2 presents the extraction of $\tau_{0}$ and derivation of the PEC signal's inverse time derivative, $|\nabla|^{-1}$, Sect. 3 showcases the lift-off independence of $|\nabla|^{-1}$ as well as the effects of permeability variations on this feature using COMSOL Multiphysics, while Sect. 4 concerns the experimental analysis. Section 5 discusses the conclusions, with remarks to the limitation of the proposed approach.

\section{Feature Extraction Techniques}

The evident benefit of PEC lies in the ability to monitor the diffusion of induced current through the tested medium. For ferromagnetic application, while this phenomenon is imperceptible when representing the PEC time-dependent signals in Cartesian graph, signals corresponding to different wall thicknesses are distinct in double-logarithmic and semilogarithmic graphs. This section describes the extraction of the conventional decay transition time, $\tau_{0}$ in doublelogarithmic domain and the inverse time derivative, $|\nabla|^{-1}$ in semi-logarithmic domain.

\subsection{Decay Transition Time, $\tau_{0}$}

The interaction of the supplied magnetic field with the test sample allows the induction of eddy current. While this induced current travels to the lower part of the test sample, it decays exponentially as soon as it reaches the end of the conductive medium.

In data analysis, a straight line is fitted to the diffusion part of a chosen reference signal by heuristically finding the middle point, while simultaneously examining if the fitted straight line is approximately equal to the diffusion curve [12]. The signal in this part exhibits a power law functional relationship of $\left.V(t)\right|_{t>\tau_{0}}=A t^{-n}$. The value of $n$ discerns the time derivative of the straight line in a double-logarithmic plot. The intersection point between the fitted line and the received signal is denoted as $\tau_{0}$, representing the estimation of the time where diffusion stops. It is then possible to determine the pipe wall thickness by comparing the value of $\tau_{0}$ of the measurement signals with that of the reference signal. Higher values of $\tau_{0}$ depict thicker metal layer, while lower values describe otherwise.

\subsection{Inverse Time Derivative, $|\nabla|^{-1}$}

The extraction of $|\nabla|^{-1}$ extends the work of Ref. [10], where the derivation starts by representing the time-dependent induced voltage in the detector coil, $V(t)$ as

$V(t)=b_{1} \exp \frac{-\pi^{2} t}{\sigma \mu d^{2}}+\sum_{i=2}^{\infty} b_{i} \exp \left(-c_{i} t\right)$

where $\sigma, \mu$, and $d$ are the conductivity, permeability and thickness of the pipe wall, respectively. The term $b_{1}, b_{i}$ and $c_{i}$ are the fitted coefficients. The term $1 / \sigma \mu d^{2}$ is the largest time constant appearing in the exponential term in Eq. (1). This, in return, becomes dominant during eddy current decay phase, enabling the expression of induced voltage in simpler terms as

$\left.V(t)\right|_{t \gg 0} \approx b_{1} \exp \frac{-\pi^{2} t}{\sigma \mu d^{2}}$

Expressing the induced voltage in its natural logarithm, Eq. (2) becomes

$\left.\ln [V(t)]\right|_{t \gg 0} \approx \ln b_{1}+\frac{-\pi^{2} t}{\sigma \mu d^{2}}$.

The induced voltage in its natural logarithm behaves like a straight line as $t \gg 0$ [10], thus the time derivative of this straight line can be obtained by

$\left.\left|\frac{\mathrm{d} \ln [V(t)]}{\mathrm{d} t}\right|\right|_{t \gg 0} \approx \frac{-\pi^{2}}{\sigma \mu d^{2}}$

Assuming homogeneous permeability across the test zone, the proportionality of the inverse of the signal's time derivative with the squared of the wall thickness can be seen

$$
\begin{aligned}
d^{2} & \left.\propto\left|\frac{\mathrm{d} t}{\mathrm{~d} \ln [V(t)]}\right|\right|_{t \gg 0} \\
& \propto|\nabla|^{-1}
\end{aligned}
$$

Although Eq. (5) suggests zero value of $|\nabla|^{-1}$ for PEC signal in air, $|\nabla|_{0}^{-1}$, the implementation in this paper extracts $|\nabla|^{-1}$ by gating the value of induced voltage within a certain range, instead of evaluating the signal during its decay phase. This is to avoid detailed process of identifying the diffusion and decay phases of the signal, while at the same 
time, extending the range of measurement. In this regard, $|\nabla|_{0}^{-1}$ does not possess the value of zero, although relatively small.

\section{Feature Analysis Using Modelling}

The multilayered structure of pipe prohibits any contact with the pipe wall, which consequently constrains the operator from getting the information on the electromagnetic properties of the pipe as well as the lift-off. In practice, PEC instruments assume the test zone to exhibit similar magnetic permeability with the reference zone [13]. Meanwhile, lift-off remains a problem as the insulation thickness is often varied due to machining and installation error. This section presents the immunity of $|\nabla|^{-1}$ towards lift-off. Moreover, the linearity of $|\nabla|^{-1}$ and thickness with varying values of permeability of carbon steel sample is also presented.

A 2D axisymmetric COMSOL Multiphysics model has been developed, which has the following parameters: excitation coil outer diameter, $d o_{e}=80 \mathrm{~mm}$, excitation coil inner diameter, $d i_{e}=58 \mathrm{~mm}$, excitation coil height, $h_{e}=$ $12.5 \mathrm{~mm}$, excitation coil offset, $O_{e}=2 \mathrm{~mm}$, sensing coil outer diameter, $d o_{s}=105 \mathrm{~mm}$, sensing coil inner diameter, $d i_{s}=83 \mathrm{~mm}$, sensing coil height, $h_{s}=12.5 \mathrm{~mm}$, and sensing coil offset, $O_{s}=2 \mathrm{~mm}$. The value of $O_{s}$ refers to the thickness of the coil former base. The supply current is set at $2 \mathrm{~A}$, while the number of turns of both excitation and sensing coil is fixed at 210 . The carbon steel test sample has the following parameters: relative permeability, $\mu=100$, and conductivity, $\sigma=5.5 \mathrm{MS} / \mathrm{m}$. The thickness, $d$ is varied from $4 \mathrm{~mm}$ to $12 \mathrm{~mm}$, at an increment of $2 \mathrm{~mm}$. The parameters chosen are based on literature, which is proven to be sufficient to penetrate through $12 \mathrm{~mm}$ thick carbon steel.

\subsection{Lift-Off Invariance}

The distance of the probe to the test sample is varied from 30 $\mathrm{mm}$ to $40 \mathrm{~mm}$, at an increment of $2 \mathrm{~mm}$, representing different levels of lift-off. The effect of varying lift-off, which can be seen in Fig. 1a, suggests the minimal lift-off influence on the time derivative of the signals. In contrast, the amplitude the signals can be observed to reduce with thicker lift-off, owing to the weakened interaction of the induced eddy current.

As previously mentioned, signal gating is carried out by gating the signal in the range of $10^{-3} V \leq V(t) \leq 10^{-4} V$, including PEC signal in air, $V_{a i r}$. Following this, $|\nabla|^{-1}$ is extracted by computing the linear fitting of the excerpts of the logarithmic PEC signals, and the approximately linear relationship of the features with their corresponding
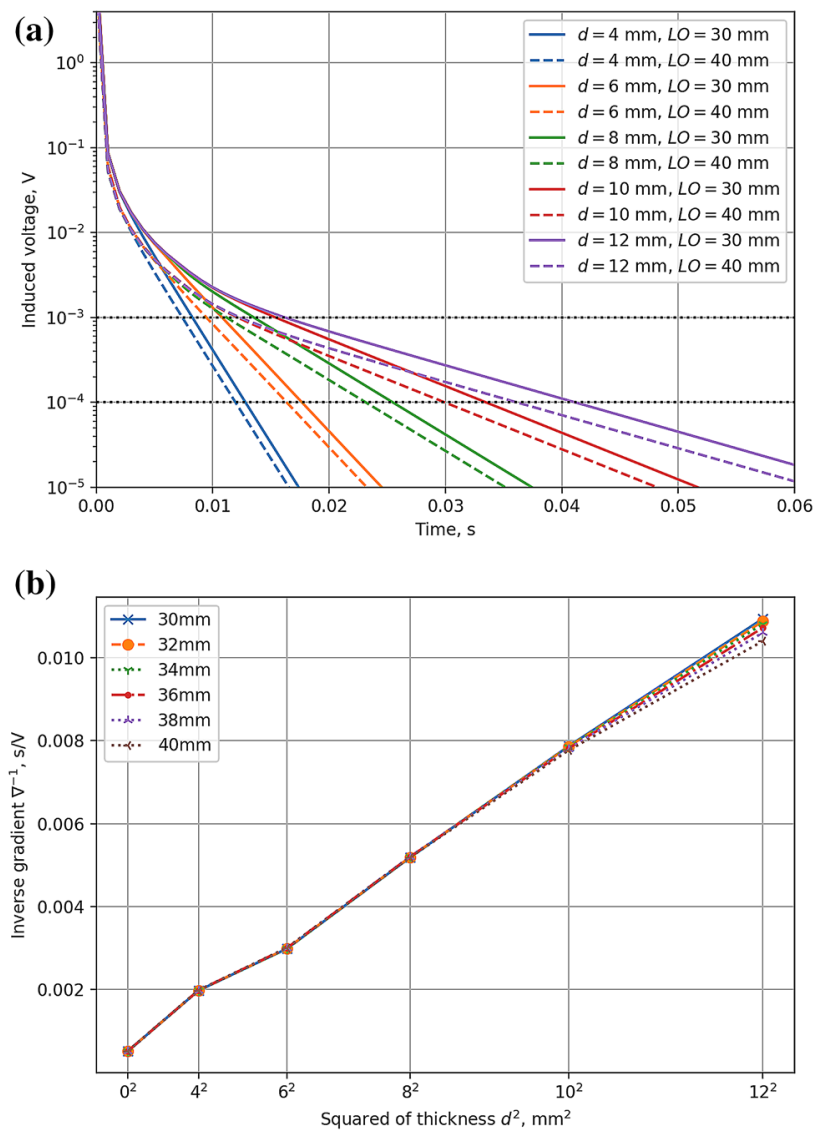

Fig. 1 (a) Simulated PEC signals with varying lift-off and sample thickness, and (b) Extracted $|\nabla|^{-1}$ corresponding to different lift-offs and thicknesses

thickness squared, $d^{2}$, as shown in Fig. 1b. It is apparent that the changes in lift-off as much as $10 \mathrm{~mm}$ has significantly small effect on $|\nabla|^{-1}$, suggesting the high potential of employing such technique in training a sophisticated fitting model.

\subsection{Variations in Permeability}

In the interest of showcasing the flexibility of using $|\nabla|^{-1}$ on different carbon steel pipes, the pipe wall's relative permeability is varied from 80 to 120 , with an increment of 10 . Figure 2a shows the signals obtained for different wall thickness, with different permeability values. The time derivatives of the signals appear to differ with different values of permeability. This consequently affects the extracted value of $|\nabla|^{-1}$, as shown in Fig. 2b. However, permeability does not affect the the linearity of the feature with respect to $d^{2}$, which proves to still be efficient across different carbon steel pipes. 


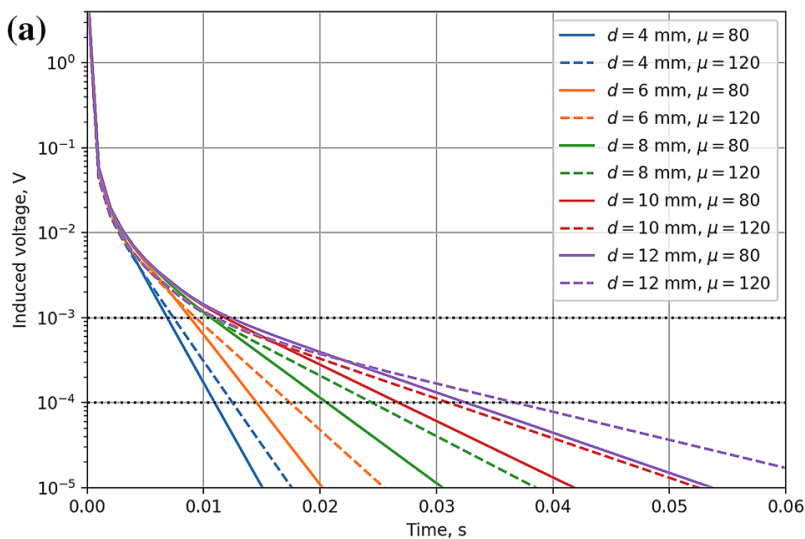

(b)

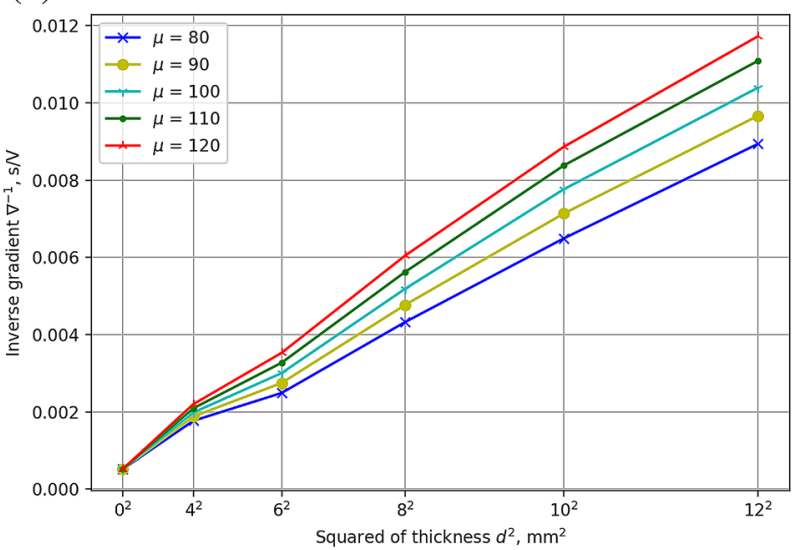

Fig. 2 a Simulated PEC signals with varying permeability and sample thickness, and $\mathbf{b}$ Extracted $|\nabla|^{-1}$ corresponding to different permeability values and thicknesses

\section{Experimental Results}

\subsection{Experimental Setup}

A transmit-receive pancake probe was developed with the same parameters as the corresponding numerical model in Sect. 3. Carbon steel plates of S275 grade were used, with thicknesses of 4-12 mm, in $2 \mathrm{~mm}$ increment. The excitation voltage was $10 \mathrm{~V}$, which supplied approximately 2 A current through the excitation coil. The current was turned on for $62.5 \mathrm{~ms}$, before being turned off for the same duration. For the purpose of this experiment, data was acquired at the rising edge, and 16 pulse signals were averaged for each test for the purpose of noise reduction. The diameter of the copper wire used for both transmit and receive coils was 0.32 $\mathrm{mm}$.

\subsection{Effects of Plate Thickness and Lift-Off}

For each thickness, lift-off was varied from 30 to $40 \mathrm{~mm}$, in 2 $\mathrm{mm}$ increment. The test was repeated five times for reasons of

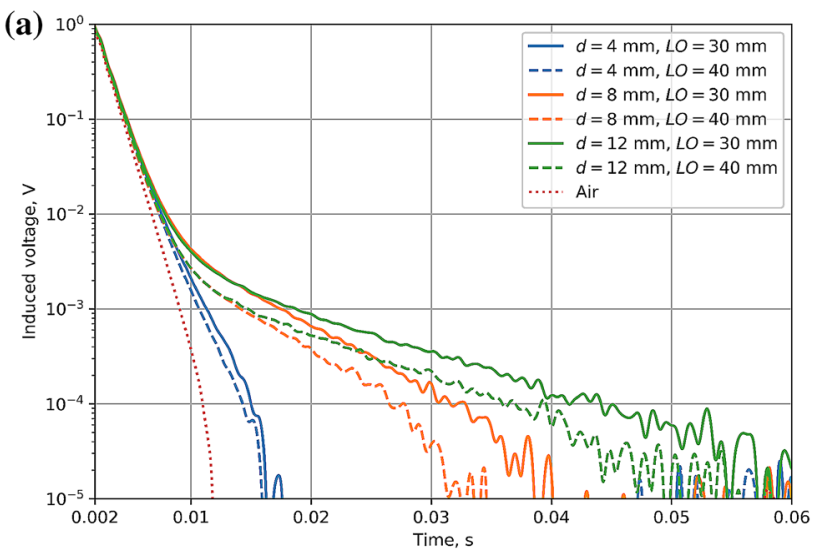

(b)

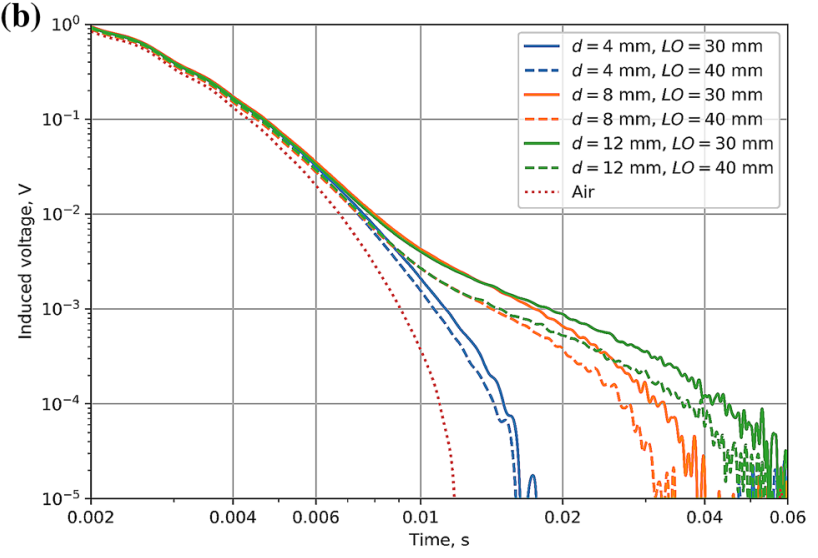

Fig. 3 PEC signals obtained for different lift-off values and thicknesses in a semi-logarithmic, and $\mathbf{b}$ double-logarithmic representation

repeatability. The signals obtained for $30 \mathrm{~mm}$ and $40 \mathrm{~mm}$ liftoff with $4 \mathrm{~mm}, 6 \mathrm{~mm}$, and $12 \mathrm{~mm}$ thicknesses, are visualised in a semi-logarithmic graph in Fig. 3a, b. The tops of the signals in the experimental setup are clipped using TVS diode to avoid overvoltage supply to the data acquisition (DAQ) card.

The effect of lift-off is seen to resemble that of the modelling results, where higher lift-off appears to scale down the amplitude of the induced voltage. Note that both Fig. 3a, b are in logarithmic representation.

Similar with the modelling results in Sect. 3, sample thickness can be observed to affect the time derivative of the induced voltage in semi-logarithmic representation. Meanwhile, in a double-logarithmic representation, thinner samples can be seen to posses faster decay transition time by examining the point in time where the PEC signal begins to break into a more pronounced downward direction, hereinafter referred to as the bending point.

\section{3 $\tau_{0}$ Extraction}

The extraction of $\tau_{0}$ was initiated with fitting a straight line to the diffusion part of a chosen PEC reference signal. In prac- 
tice, this is commonly done by heuristically finding the range of amplitude to be fitted, while simultaneously examining if the fitted straight line is approximately equal to the diffusion curve.

In double-logarithmic domain, the power law curve of the induced voltage during diffusion can be represented as $\log _{10}[V(t)]=\log _{10}(A)-n \log _{10}(t)$, where $n$ is the the slope of the PEC signal (in logarithmic scale). This explains the straight line-like behaviour during the diffusion phase, which serves as a reference for both $\tau_{0}$ extraction and normalisation. To effectively find this straight line, bi-symmetric logarithmic transformation was carried out on each PEC signal, using

$$
V^{b i-\operatorname{symlog}}(t)=\operatorname{sgn}[V(t)] \cdot \log _{10}\left[1+\mid\left(V(t) \mid / 10^{C}\right],\right.
$$

where $C$ is a scaling constant to smoothly transform the region near zero so that it remains finite. In this case, $C$ was chosen to be -4.5 , which equals to the lowest order observed in the acquired signals.

This bi-symmetric transformation was utilised instead of conventional logarithmic transformation to avoid negative values in the signals to be treated as infinite, which allows the analysis of noise margin and probe vibration. Another reason for using this transformation was to enable signal normalisation to be carried out on signals affected by lift-off variations. Suppressing the lift-off effects on the affected signals, after being bi-symmetrically transformed, can be conveniently achieved by arithmetically shifting each affected signal to their corresponding reference signal.

To develop the reference line, a PEC signal for $12 \mathrm{~mm}$ thick sample with $40 \mathrm{~mm}$ lift-off, $V_{12 \mathrm{~mm}, 40 \mathrm{~mm}}^{\text {bi-symlog }}(t)$, was used as a reference signal. The induced voltage in the range of $0.0130 \mathrm{~s} \leq t \leq 0.0175 \mathrm{~s}$ was extracted, and a straight line of $V^{b i-\operatorname{sym} l o g}(t)=a \log _{10}(t)+b$ was fitted. The amplitude of the reference line was then arithmetically reduced to $93 \%$ and extrapolated, in order to allow establishing the crossing point between the shifted reference line with the PEC signals.

However, as seen in Fig. 4, the $4 \mathrm{~mm}$ and $6 \mathrm{~mm}$ signals deviated earlier than $0.013 \mathrm{~s}$, which caused the extrapolated fitted line to fail in representing the bending point of these signals. In this regard, the extrapolated values of the fitting line for $t \leq 0.013 \mathrm{~s}$ were replaced with the arithmetically reduced $V_{12 \text { mm, } 40 \mathrm{~mm}}^{b i}(t)$ signal. The new reference line is denoted by the dashed line in Fig. 4, while the time for the PEC signals to cross the reference line is extracted as $\tau_{0}$.

Normalisation was imposed on all PEC signals to suppress the lift-off effects. This was achieved by subtracting each signal with the difference in the signal value at $t=0.0130 \mathrm{~s}$ with $V_{12 \mathrm{~mm}, 40 \mathrm{~mm}}^{\text {bi-symlog }}(0.0130 \mathrm{~s})$. The effect of this normalisation procedure is shown in Fig. 5.

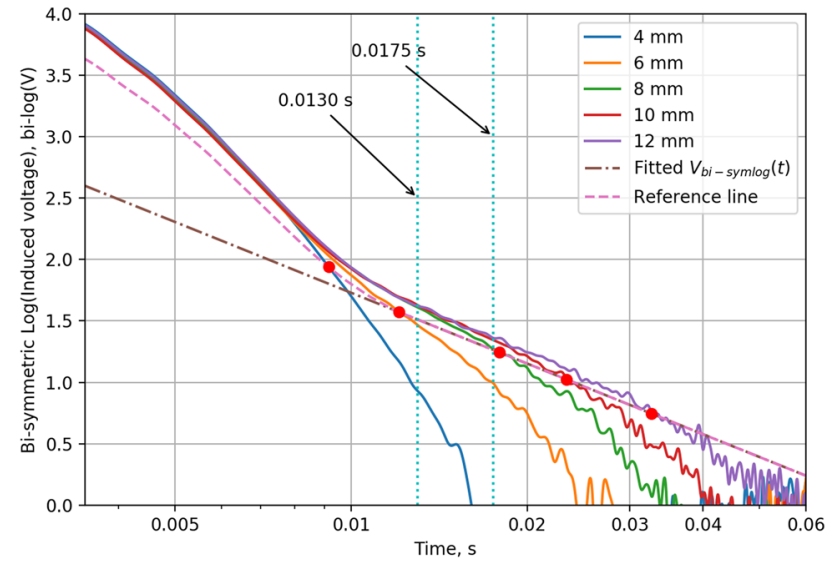

Fig. 4 Visualisation of reference line and PEC signals

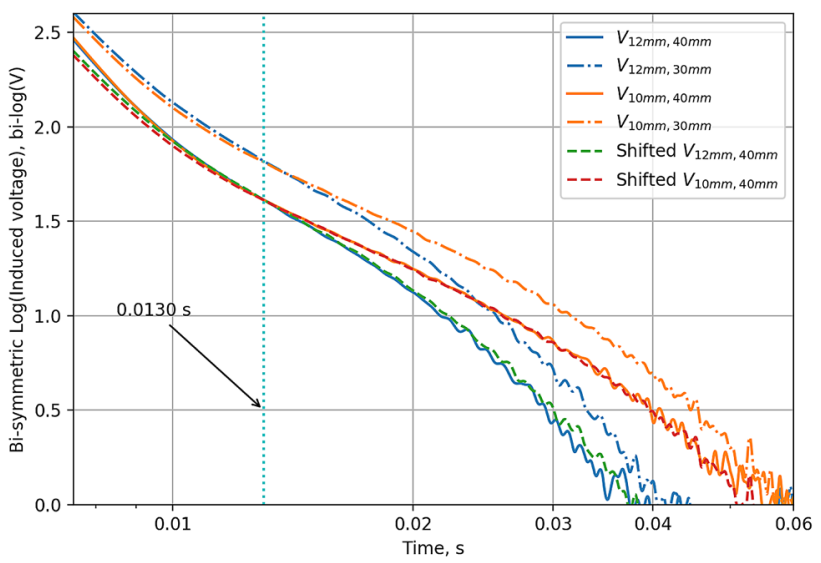

Fig. 5 Visualisation of normalised and non-normalised signals

\section{$4.4|\nabla|^{-1}$ Extraction}

As $|\nabla|^{-1}$ extraction was done in semi-logarithmic domain, it did not require the process of transforming the signals with bi-symmetric logarithmic procedure. Instead, the excerpts of the induced signals (induced voltage within the range of $\left.10^{-4} \mathrm{~V} \leq V(t) \leq 10^{-3} \mathrm{~V}\right)$ were fitted with the equation $\ln [V(t)]=\nabla(t)+\lambda$. The absolute value of the reciprocal of $\nabla$ equals to the value of $|\nabla|^{-1}$.

\subsection{Calibration Process}

Having discussed the extraction of both $\tau_{0}$ and $|\nabla|^{-1}$, it is now possible to establish the procedures to enable in-situ calibration for PEC instrument. In a real-life deployment of a PEC instrument, nominal thickness signal (in this case, PEC signal for $12 \mathrm{~mm}$ ) can be obtained by choosing single or multiple signals in the inspected area which possesses the highest feature measurement as well as having the least variation in the $\mathrm{C}$-scan. The extracted $\tau_{0}$ from this signal can 

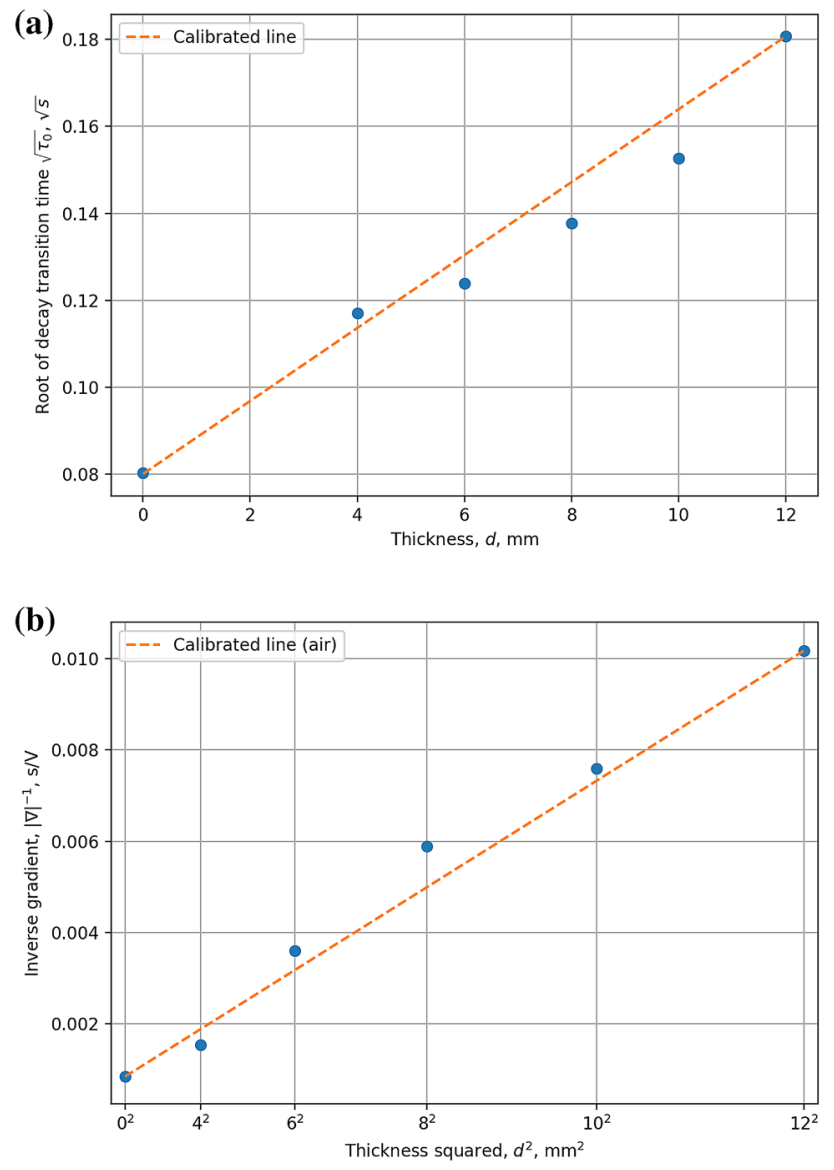

Fig. 6 Calibration curve for $\mathbf{a} \tau_{0}$, and $\mathbf{b}|\nabla|^{-1}$

then be compared with the $\tau_{0 \text {,air, }}$, which is stored in the computational memory. A straight line is drawn crossing $\sqrt{\tau_{0 \text {,air }}}$ and $\sqrt{\tau_{0, \text { nominal }}}$ in the graph of $\sqrt{\tau_{0}}$ against wall thickness, $d$, as demonstrated in Fig. 6a. The values of $\sqrt{\tau_{0}}$ obtained for thicknesses thinner than the $12 \mathrm{~mm}$, can be directly evaluated based on the calibration curve.

Similar to the approach taken to establish the calibration curve for $\tau_{0}$, a straight line is drawn passing through $|\nabla|_{\text {air }}^{-1}$ and $|\nabla|_{\text {reference, }}^{-1}$, on the graph of $|\nabla|^{-1}$ against $d^{2}$, as shown in Fig. 6b. The information regarding the straight line, i.e. the gradient and the y-intercept, is then used to infer the estimated thickness by evaluating the obtained $|\nabla|^{-1}$ values.

\subsection{Performance Analysis}

In terms of signal processing, the $|\nabla|^{-1}$ technique does not require normalisation process, while $\tau_{0}$ technique does. While lift-off contributes to the change in the signal amplitude, the decay rate is still maintained. However, as $\tau_{0}$ technique makes use of the intersection point between the signal with the reference line, signal conditioning is required to redress this change in the signal amplitude. This contributes to more configuration parameters to be determined, which further challenges the inspection process to be more automated.

Figure 7 shows the comparisons of predicted thickness based on different feature extraction techniques, applied on PEC signals with different lift-offs and thicknesses. The most noticeable observation is that across different values of liftoffs, the estimations for $6 \mathrm{~mm}, 8 \mathrm{~mm}$ and $10 \mathrm{~mm}$ samples by using $\tau_{0}$ tend to fall below the estimation line, while this is the opposite for $|\nabla|^{-1}$ feature. Undoubtedly, estimations from both features can be used to effectively provide a more accurate measurement. The spread in $\tau_{0}$ estimation for thinner samples looks to be tighter, as compared to thicker samples. This is expected, as the extraction of $\tau_{0}$ requires finding the crossing point of the PEC signals with the reference line, which is challenged by the presence of noise in signals of thicker samples. Unlike $\tau_{0},|\nabla|^{-1}$ has a relatively similar spread across different thicknesses and lift-offs, making the measurement to be more repeatable and robust to noise.

Further investigation should provide a better analysis to demonstrate the independence (or lack thereof) of the two features with each other. This should also open up the opportunity to further explore the possibilities of combining the information provided by each feature using sophisticated feature fusion approaches.

Figure 8 depicts the collective root-mean-square-error (RMSE) values for both features, represented by their respective lift-off and sample thickness. As noted, on average, the performance of $|\nabla|^{-1}$ was better than $\tau_{0}$. Lift-off can be claimed to have insignificant influence on both feature performances where the RMSE values are almost consistent across all lift-off values. Although in terms of lift-off variation, the RMSE value for $|\nabla|^{-1}$ is lower than $\tau_{0}$, it is noticeable that this is not the case for thickness variation.

Although $|\nabla|^{-1}$ has a lower RMSE across different sample thicknesses despite the lift-off variation, this is not the case particularly for $4 \mathrm{~mm}$ thick plate. This can be attributed to the deficient fitting of the feature around the calibration curve at $4 \mathrm{~mm}$ thickness. Nevertheless, estimating thickness using $|\nabla|^{-1}$ is still significantly better for $10 \mathrm{~mm}$ and $12 \mathrm{~mm}$ samples, and the overall RMSE for $|\nabla|^{-1}$ was recorded to be $0.67 \mathrm{~mm}$, outperforming $\tau_{0}$ by $0.13 \mathrm{~mm}$.

\section{Conclusions}

This paper has presented an in-situ calibration procedure using $|\nabla|^{-1}$ feature, aimed to be applied in carbon steel pipe inspection. The amount of corrosion, represented as different plate thicknesses in the developed experiment, can be determined by calibrating the reference PEC signal with the PEC signal in air. The linear relationship between the extracted features, $\tau_{0}$ and $|\nabla|^{-1}$, has been described using both analytical solutions and the underlying physical phenomenon. 
Fig. 7 Estimated thickness against real thickness for different lift-off
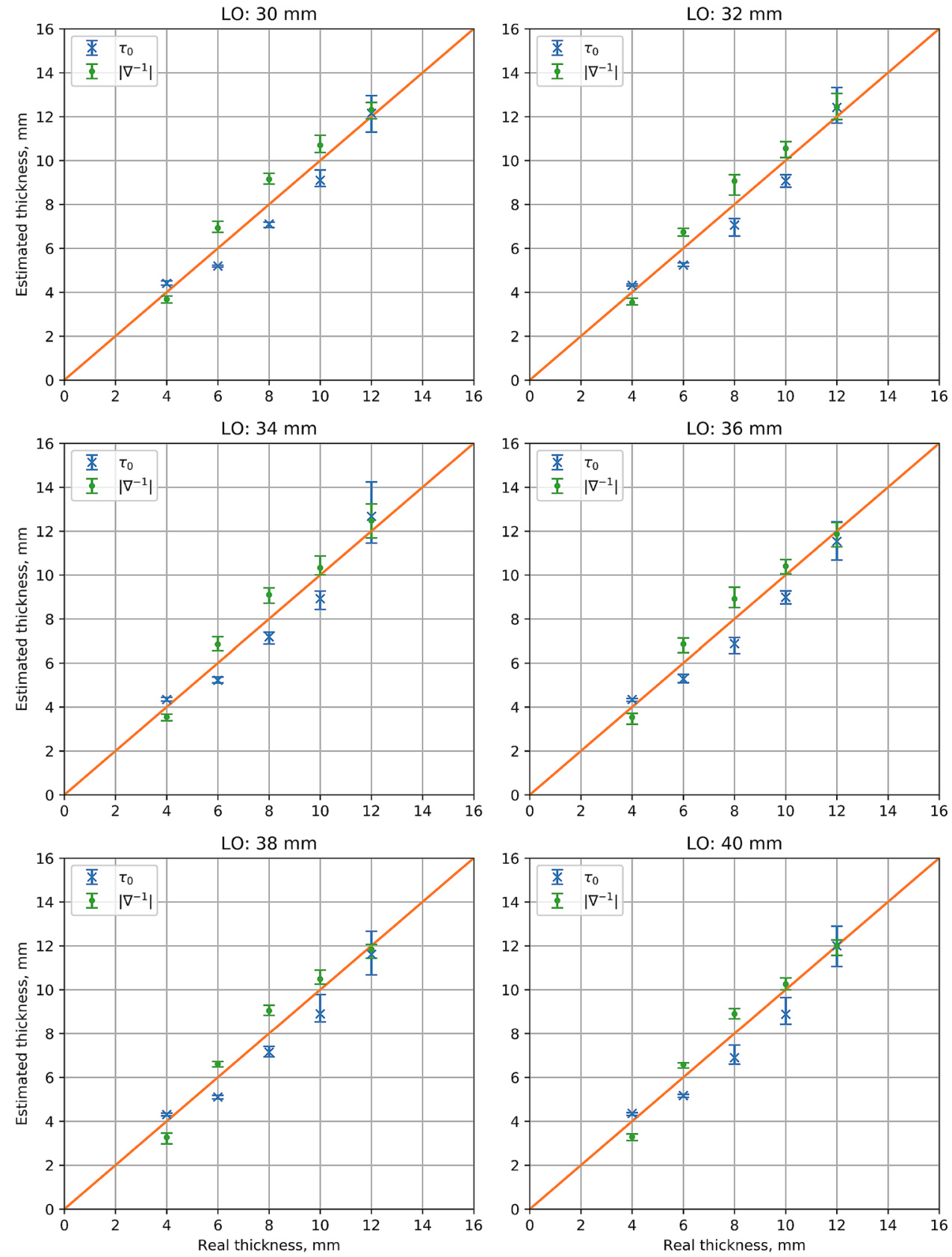

Normalisation procedure has been discussed, which enables $\tau_{0}$ immunity to changes in lift-off. The independence of signal's decay rate on lift-off gives $|\nabla|^{-1}$ technique an advantage over $\tau_{0}$, where extracting $|\nabla|^{-1}$ requires no signal conditioning. Experimental evaluation suggests the superiority of $|\nabla|^{-1}$ over $\tau_{0}$ in handling low signal-to-noise ratio (SNR) for thick samples, while also outperforms $\tau_{0}$ in terms of thickness estimation.

The experimental analysis has been carried out for $4 \mathrm{~mm}$ $\leq$ thickness $\leq 12 \mathrm{~mm}$ plate samples, while the lift-off has been varied in the range of $30 \mathrm{~mm} \leq$ lift-off $\leq 40 \mathrm{~mm}$. While this is applicable to most pipe dimensions, it is still appealing to investigate the linearity of the proposed techniques in other range of measurements, particularly up to $65 \mathrm{~mm}$ sample thickness, as suggested in the ISO 20663:2017(E). Probe parameters are also expected to be tuned in order to maintain the linear relationship of the features with the thickness, for different range of lift-offs and thicknesses. Upcoming work should also include carrying out the study on pipes to incorporate the effects of pipe's curvature on the linearity of $|\nabla|^{-1}$ with respect to $d^{2}$. 
Fig. 8 RMSE comparisons for $\tau_{0}$ and $|\nabla|^{-1}$ in terms of lift-off and sample thickness

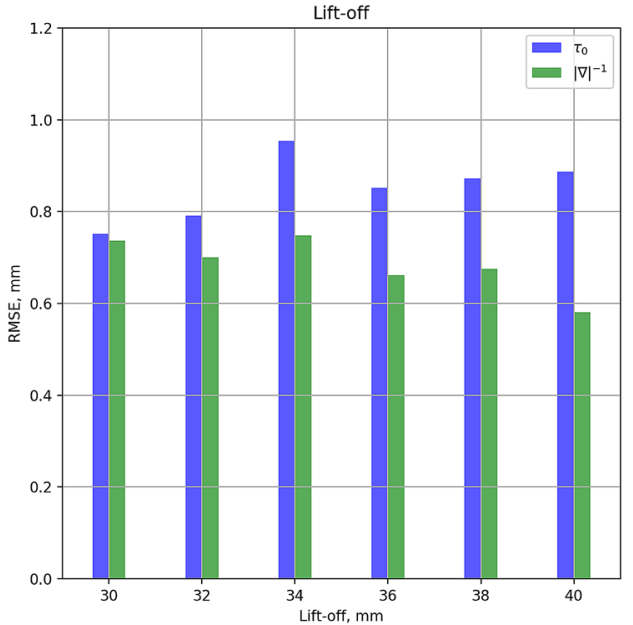

Acknowledgements The authors would like to thank Lloyd's Register Foundation for funding of this research. Lloyd's Register Foundation helps to protect life and property by supporting engineering-related eduction, public engagement and the application of research.

Open Access This article is licensed under a Creative Commons Attribution 4.0 International License, which permits use, sharing, adaptation, distribution and reproduction in any medium or format, as long as you give appropriate credit to the original author(s) and the source, provide a link to the Creative Commons licence, and indicate if changes were made. The images or other third party material in this article are included in the article's Creative Commons licence, unless indicated otherwise in a credit line to the material. If material is not included in the article's Creative Commons licence and your intended use is not permitted by statutory regulation or exceeds the permitted use, you will need to obtain permission directly from the copyright holder. To view a copy of this licence, visit http://creativecomm ons.org/licenses/by/4.0/.

\section{References}

1. van den Berg, S.: Modelling and Inversion of Pulsed Eddy Current Data [Dissertation]. Delft University of Technology, Delft (2003)

2. De Haan, V.O., De Jong, P.A.: Simultaneous measurement of material properties and thickness of carbon steel plates using pulsed eddy currents. In: 16th World Conference on Non-Destructive Testing, Aug 30-Sep 3, 2004, Montreal, Canada (2004)

3. Eddy, I.C., Underhill, P.R., Morelli, J., et al.: Pulsed eddy current response to general corrosion in concrete bar. In: 2019 NDT in Canada, June 18-20, Alberta, Canada (2019)

4. Xu, Z., Wu, X., Li, J., et al.: Assessment of wall thinning in insulated ferromagnetic pipes using the time-to-peak of differential pulsed eddy-current testing signals. NDT \& E Int. 51, 24-29 (2012)

5. Huang, C., Wu, X., Xu, Z., et al.: Ferromagnetic material pulsed eddy current testing signal modeling by equivalent multiple-coilcoupling approach. NDT \& E Int. 44, 163-168 (2011)
6. Ulapane, N., Nguyen, L., Miro, J.V., et al.: Designing a pulsed eddy current sensing set-up for cast iron thickness assessment. In: Proceedings of the 2017 12th IEEE Conference on Industrial Electronics and Applications, ICIEA 2017, pp. 901-906 (2018)

7. Ulapane, N., Alempijevic, A., Calleja, T.V., et al.: Pulsed eddy current sensing for critical pipe condition assessment. Sensors 17, 2208-2233 (2017)

8. Ulapane, N., Nguyen, L.: Review of pulsed-eddy-current signal feature-extraction methods for conductive ferromagnetic materialthickness quantification. Electronics 8, 470-482 (2019)

9. Buck, J.A., Kramer, C., Lei, J., et al.: Field implementation of a transient eddy current system for carbon steel pipe thickness measurements. In: 6th International CANDU In-Service Inspection Workshop, November 15-17, 2016, Ontario, Canada (2016)

10. Ulapane, N., Alempijevic, A., Miro, J.V., et al.: Non-destructive evaluation of ferromagnetic material thickness using pulsed eddy current sensor detector coil voltage decay rate. NDT \& E Int. 100, 108-114 (2018)

11. Huang, C., Wu, X.: An improved ferromagnetic material pulsed eddy current testing signal processing method based on numerical cumulative integration. NDT \& E Int. 69, 35-39 (2015)

12. Kitane, Y., Ando, S., Itoh, Y., et al.: Numerical study on pulsed eddy current thickness measurement for steel plates with thickness loss. In: Proceedings of the Fourteenth East Asia-Pacific Conference on Structural Engineering and Construction, pp. 1-8 (2016)

13. 20669:2017(E) I. Non-destructve testing-pulsed eddy current testing of ferromagnetic metallic components. International Organization for Standardization, Geneva (2017)

Publisher's Note Springer Nature remains neutral with regard to jurisdictional claims in published maps and institutional affiliations. 